Document downloaded from:

http://hdl.handle.net/10251/171702

This paper must be cited as:

Samaniego, D.; Vidal Rodriguez, B. (2020). Brillouin wavelength-selective all-optical polarization conversion. Photonics Research. 8(4):440-447.

https://doi.org/10.1364/PRJ.371513

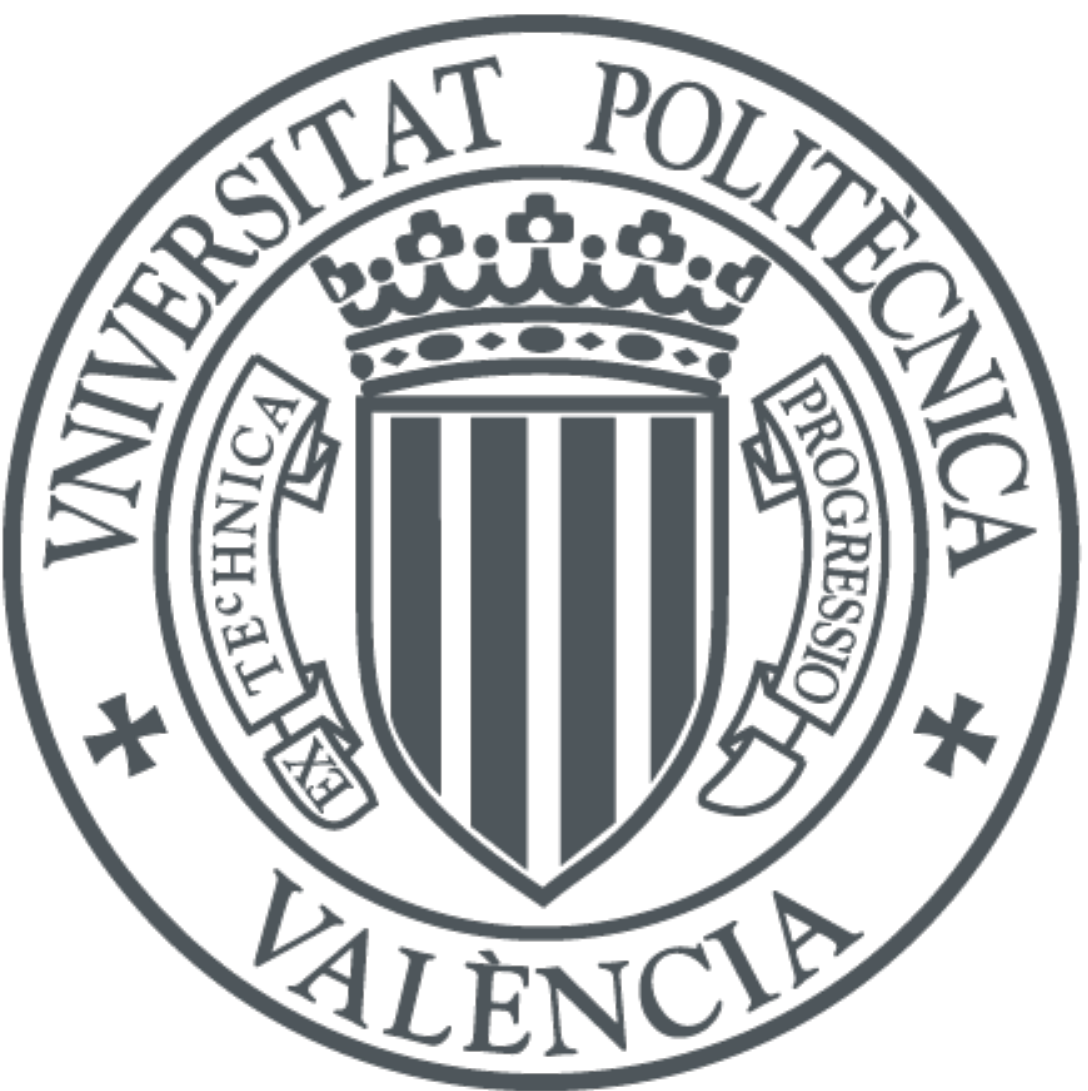

The final publication is available at

https://doi.org/10.1364/PRJ.371513

Copyright Optical Society of America

Additional Information 


\title{
Brillouin wavelength-selective all-optical polarization conversion
}

\author{
Diego Samaniego, Borja Vidal, * \\ ${ }^{I}$ Nanophotonics Technology Center, Universitat Politecnica de Valencia, c/ Camino de Vera, sn, \\ Valencia, 46022, Valencia, Spain \\ *bvidal@dcom.upv.es
}

\begin{abstract}
The manipulation of the polarization properties of light in guided media is crucial in many classical and quantum optical systems. However, the capability of current technology to finely define the state of polarization of particular wavelengths is far from the level of maturity in amplitude control. Here, we introduce a light-by-light polarization control mechanism with wavelength selectivity based on the change of the phase retardance by means of stimulated Brillouin scattering. Experiments show that any point on the Poincaré sphere can be reached from an arbitrary input state of polarization with little variation of the signal amplitude $(<2.5$ $\mathrm{dB})$. Unlike other Brillouin processing schemes, the degradation of the noise figure is small ( $1.5 \mathrm{~dB}$ for a full $2 \pi$ rotation). This all-optical polarization controller can forge the development of new polarization-based techniques in optical communication, laser engineering, sensing, quantum systems and light-based probing of chemical and biological systems.
\end{abstract}

(c) 2019 Optical Society of America under the terms of the OSA Open Access Publishing Agreement

\section{Introduction}

The ability to manage the state of polarization (SOP) is essential in controlling many polarization-related phenomena in different areas of photonics [1-2]. New tools and techniques have evolved from advanced control of light polarization. In free-space optics, the development of polarization structured beams has opened new paths in both scientific and engineering applications, for example, spatially-dependent polarization beams for optical tweezers [3]. However, in optical fibers and integrated waveguides there are no tools offering similar potential for polarization structuring.

Polarization management in optical fibers is usually performed by means of: 1) multiple stages with fixed linear retardations and variable orientation angles, typically implemented using cascaded mechanically-controlled stress-induced birefringence in rotatable waveplates or electro-optical devices exploiting the Pockels effect [4-5]; 2) a single stage with both variable linear retardation and orientation, implemented using fiber implementations of a Babinet-Soleil compensator [6]; 3) multiple stages with variable retardation and fixed orientation [7]. However, all these are broadband devices and they cannot provide wavelength-selective polarization control analogous to the granularity that can be achieved in spatial dimensions with SLMs.

Nonlinear effects in optical fibers have been proposed to implement different kinds of polarizers through the mechanism known as polarization attraction. The polarization sensitive gain of nonlinear effects such as Stimulated Brillouin Scattering (SBS) [8-10], Stimulated Raman Scattering (SRS) [11] and parametric amplification [12] can be used to implement a functionality similar to a polarizer but usually providing polarization-dependent gain instead of loss. A second class of polarizers enabling energy conservation, known as lossless polarizers [13-18], can also be achieved exploiting cross-polarization modulation but they need rather strong optical powers, typically in the order of Watts, and the efficiency of the attractor depends on the pump SOP. 
Here, we introduce a new nonlinear optical functionality, complete polarization control. Instead of the implementation of a polarizer, we report an all-optical analogue of a polarization controller based on a variable linear retarder (such as a wave plate) with fixed orientation plus one variable circular retarder (optical rotator). The variable retarders rely on exploiting the capability of Brillouin scattering to induce arbitrary birefringence in optical fibers to control the phase shift between orthogonal components of the SOP while conserving the energy of the signal though a combination of phase shifting and gain/loss compensation.

\section{Nonlinear polarization control}

Since Brillouin scattering is originated from an interference between counterpropagating waves through electrostriction, its efficiency is polarization dependent, i.e. the gain (or loss) coefficient is maximum when the pump and signal electric fields rotate describing the same ellipses with opposite senses. From this polarization dependence, a controlled phase shift between eigenmodes can be introduced using SBS if the pump is aligned with one eigenmode of the signal. Thus, SBS can induce an optically-controlled phase retardance between linearlypolarized eigenmodes in a guided medium such as optical fiber. This polarization control is wavelength selective since the Brillouin response bandwidth can be tuned from $30 \mathrm{MHz}$ to tens of $\mathrm{GHz}$ due to the short coherence lifetime of hypersonic phonons in fiber. It allows independent control of the SOP of individual optical channels in DWDM scenarios.

The natural response of SBS associates a phase shift with a gain (Stokes) or a loss (antiStokes) response, as shown in the inset of Figure 1a (blue solid/dotted curves). However, it is possible to combine Brillouin gain and loss resonances to compensate each other in magnitude while adding the phase shifts provided by both responses, as shown the inset in Figure 1a (green curve). This combination of two pump waves can easily be achieved by biasing an external modulator at the minimum bias point (MITB) with a microwave signal of frequency $f_{p}$. The maximum phase shift is obtained when $f_{p}=v_{B} \pm \frac{\Delta v_{B}}{2}$, where $v_{B}$ is the Brillouin frequency shift and $\Delta v_{B}$ is the FWHM bandwidth of the Brillouin gain response. This approach allows the enhancement of the phase response while maintaining the amplitude of the signal minimally affected, as shown in Figure 1b. It is analogous to an optical all-pass filter, which have been proposed to control the phase of optically-modulated RF signals [19-20].

From this polarization dependence of the Brillouin all-pass filter response a polarization controller can be built. In particular, full polarization control can be achieved using SBS to implement two variable retarders with fixed orientations, as shown in Figure 1 (a). Brillouin allows the implementation of arbitrary birefringent elements with dynamic control. The pump SOP defines the birefringence type, i.e. the plane of polarization rotation on the Poincaré sphere, and the pump power controls the phase retardance $(\Gamma)$ between orthogonal polarization eigenmodes, i.e. the angle of rotation on this plane. To perform a circular retarder (Pump \#1), the pump polarization has to be circular. In Figure $1 \mathrm{c}_{2}$ it is shown how a right-handed circular polarization rotates the signal SOP clockwise. A left-handed circular pump would induce a counterclockwise rotation. On the other hand, the second stage, with Pump \#2 having a linear horizontal SOP, performs a linear retarder, as shown in Figure 1d. 
a
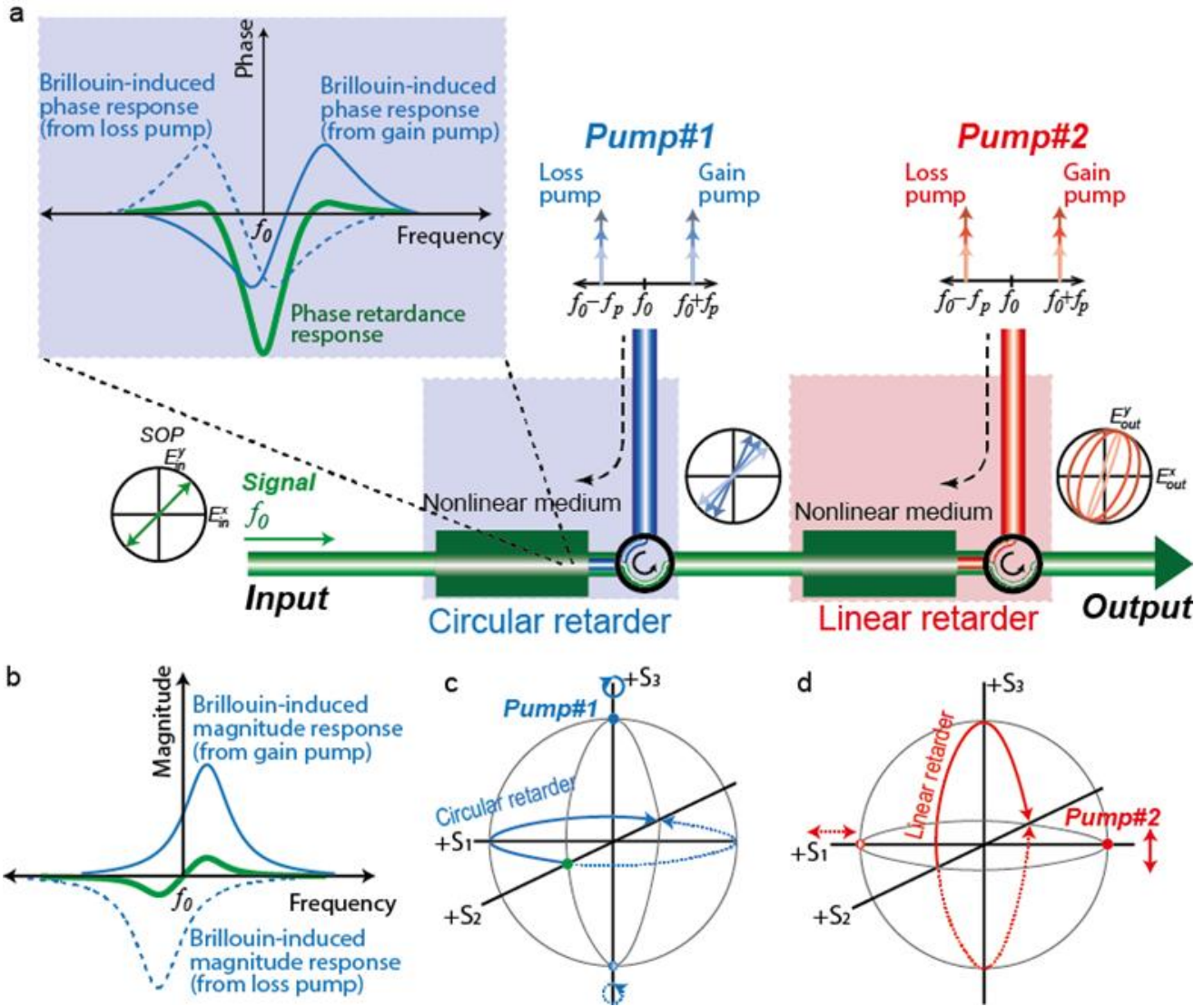

Fig. 1. (a) Concept of the nonlinear polarization controller based on two independently controlled variable elements: a circular retarder and a linear retarder. The SOP of an incoming optical signal at $f_{0}$ with, for example, linear polarization at $45^{\circ}\left(\right.$ Jones vector $\left.\left([1 / \sqrt{2}(1,1)]^{T}\right)\right)$ is altered by a pair of pump signals, gain and loss, respectively, at $f_{0}-f_{p}$ and $f_{0}+f_{p}$ with circular polarization for the circular retarder, and a second pair of pump at $f_{0}-f_{p}$ and $f_{0}+f_{p}$ with linear polarization for the linear retarder. Inset: Phase response of the all-optical polarization-dependent SBS all-pass filter (b) Magnitude of the frequency response of the alloptical polarization-dependent SBS all-pass filter. Combined gain+loss response (green), natural SBS gain response (blue solid) and loss response (blue dotted). (c) Polarization rotation on the Poincaré sphere for the ideal circular retarder. (d) Polarization rotation on the Poincaré sphere for the ideal linear retarder.

If the Brillouin gain parameter, $G=\zeta g_{0} P_{p} L /\left(A_{e f f}\right)$, is high, a significant amount of spontaneous Brillouin noise might be generated. However, this contribution can be considerably reduced if two pump pairs are used. Each orthogonal pump wave must induce a rotation in the same direction, i.e. they need to introduce an inverted Brillouin phase shift. It can be done having pumps with slightly different frequencies. One pump pair will have pump signals at frequencies $f_{0} \pm f_{p_{1}}$ and the second pump pair with orthogonal SOP will have, $f_{0} \pm$ $f_{p_{2}}$, where $f_{p 1}=v_{B}+\Delta v_{B} / 2$ and $f_{p 2}=v_{B}-\Delta v_{B} / 2$. This doubles the total retardance induced over the signal while suppressing the generation of spontaneous Brillouin noise.

The Brillouin-induced retardance can be calculated, taking into account that SBS in low birefringence single-mode fibers can be viewed as a polarization-dependent gain (loss), where there are two orthogonal input SOPs, each one showing a different gain (loss) [8],[21]. The gain of the input SOP with the maximum gain coefficient is twice the minimum one, being $2 / 3$ 
and $1 / 3$ of the gain in a fiber with no birefringence. Thus, in a birefringent fiber, the Brillouin induced phase shift is given by,

$$
\varphi_{\max / \min }=\frac{\zeta g_{0} P_{p} L_{e f f}}{2 A_{e f f}} \frac{\Delta v_{B}\left(f_{p}-v_{B}\right)}{\left(f_{p}-v_{B}\right)^{2}+\left(\frac{\Delta v_{B}}{2}\right)^{2}}
$$

where $P_{p}$ is the pump power, $g_{0}$ is the Brillouin gain factor, $A_{e f f}$ is the effective area, $L_{e f f}$ is the effective interaction length of the fiber, and the term $\zeta$ is $2 / 3$ for the maximum gain/loss and $1 / 3$ for the minimum gain/loss in birefringent fiber. Therefore, the maximum phase retardance induced by the SBS phase shift (1) in a birefringent fiber is,

$$
\Gamma=2 \varphi_{\max }-2 \varphi_{\min }=\frac{g_{0} P_{p} L}{3 A_{e f f}} \frac{\Delta v_{B}\left(f_{p}-v_{B}\right)}{\left(f_{p}-v_{B}\right)^{2}+\left(\frac{\Delta v_{B}}{2}\right)^{2}}
$$

It can be seen in (2) that, despite the combination of gain and loss phase responses, fiber birefringence reduces the maximum phase retardance that can be induced by using SBS to the phase shift induced by a single SBS gain response in a nonbirefringent fiber. Therefore, the Jones matrix of the nonlinear polarization controller is,

$$
\begin{aligned}
& J_{S B S}= M_{2} M_{1} \\
&=\left(\begin{array}{cc}
G_{\min } A_{\min } \cdot\left(\cos \left(\frac{\Gamma_{2}}{2}\right)-j \sin \left(\frac{\Gamma_{2}}{2}\right) \cdot \cos (2 \delta)\right) & G_{\max } A_{\max } \cdot \mathrm{j} \sin \left(\frac{\Gamma_{2}}{2}\right) \cdot \sin (2 \delta) \\
G_{\min } A_{\min \cdot} \cdot \mathrm{j} \sin \left(\frac{\Gamma_{2}}{2}\right) \cdot \sin (2 \delta) & G_{\max } A_{\max } \cdot\left(\cos \left(\frac{\Gamma_{2}}{2}\right)-j \sin \left(\frac{\Gamma_{2}}{2}\right) \cdot \cos (2 \delta)\right)
\end{array}\right) \\
&\left(\begin{array}{cc}
G_{\min } A_{\min } \cos \left(\Gamma_{1} / 2\right) & G_{\max } A_{\max } \sin \left(\Gamma_{1} / 2\right) \\
-G_{\min } A_{\min } \sin \left(\Gamma_{1} / 2\right) & G_{\max } A_{\max } \cos \left(\Gamma_{1} / 2\right)
\end{array}\right)
\end{aligned}
$$

where Jones matrices, $M_{1}$ and $M_{2}$ correspond to the linear and circular retarder, respectively. $\Gamma_{1}$ is the retardance induced by circular birefringence (Pump \#1) and $\Gamma_{2}$ by the linear birefringence (Pump \#2). $\delta$ is the azimuth angle of the fast axis of the linear birefringence defined by the SOP of Pump \#2.

If the gain and loss amplitude responses perfectly compensate each other, i.e. $\left|G_{\max }\right| \cdot$ $\left|A_{\max }\right| \cong 1$, and $\left|G_{\min }\right| \cdot\left|A_{\min }\right| \cong 1$, polarization control could be achieved while preserving the energy of the incoming signal.

The system shown in Figure 2 allows the rotation to an arbitrary output SOP by the combination of two pump pairs with orthogonal polarizations for each wave retarder. Each pump pair introduces a retardance up to $\pm \pi$ in the Stokes space.

For the circular retarder, if the pump at $f_{p 1}$ has a right circular SOP, the signal SOP rotates to the West, as shown in the inset of Figure 2 (blue solid arrow). The signal SOP can be rotated to the East as show in Figure 2 (blue dotted arrow) if frequencies $f_{p 1}$ and $f_{p 2}$ are swapped, such that $f_{p 1}=v_{B}-\Delta v_{B} / 2$ and $f_{p 2}=v_{B}+\Delta v_{B} / 2$. For the linear retarder, the rotation is obtained in the same way but the SOPs of the pump waves are linear at $90^{\circ}$ and $0^{\circ}$ for $f_{p 1}$ and $f_{p 2}$, respectively. 


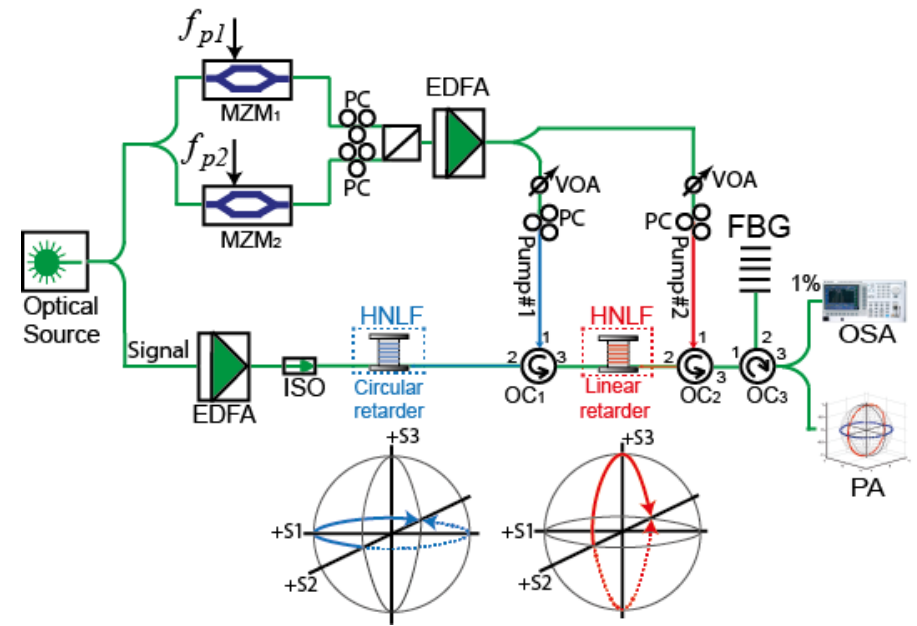

Fig. 2. Block diagram of a nonlinear all-optical polarization controller made of one circular retarder plus one linear retarder. HLNF: Highly nonlinear fiber. OC: Optical circulator; ISO: Isolator. VOA: Variable Optical Attenuator. PC polarization controller. FBG: Fiber Bragg grating. OSA: Optical spectrum analyzer. PA: Polarization analyzer.

The polarization modulation speed of the system is restricted by the propagation velocity in the nonlinear medium. For a fiber length of $1 \mathrm{~km}$, the system will have a polarization modulation speed of approximately $200 \mathrm{kHz}$. However, the use of chalcogenide fiber [22] or photonic integrated devices [23], would enhance considerably the polarization modulation bandwidth up to the $\mathrm{GHz}$ range.

\section{Results and discussion}

A theoretical model of the induced retardance by Brillouin polarization rotation has been developed from SBS equations. It is based on a theoretical analysis of the steady state stimulated Brillouin scattering in a single-mode fiber [24]. The model assumes pump depletion but neglects fiber attenuation and takes into account the vector behavior of Brillouin gain in a birefringent fiber. Theoretical estimations have been made assuming a highly nonlinear fiber (HNLF) with $A_{\text {eff }}=11 \mu \mathrm{m}^{2}, g_{0}=7.19 \times 10^{-12} \mathrm{~m} \cdot W^{-1}, \Delta v_{B}=50 \mathrm{MHz}, v_{B}=9.642 \mathrm{GHz}$, signal power at input fiber $P s=-8 \mathrm{dBm}$ and the wavelength of operation is $1548 \mathrm{~nm}$.

The experimental setup used to demonstrate the feasibility of nonlinear polarization control using SBS is the one shown in Figure 2. In particular, two Brillouin polarization control stages are implemented: a circular and a linear retarder. An optical signal is split into two paths using a fiber coupler: the upper one is used to generate the pump waves while the lower one experiences polarization rotation. The pump is generated by two x-cut Mach-Zehnder modulators $\left(\mathrm{MZM}_{1}\right.$ and $\left.\mathrm{MZM}_{2}\right)$ at minimum transmission bias (MITB) and fed by two microwave oscillators with frequencies $f_{p 1}(9.607 \mathrm{GHz})$ and $f_{p 2}(9.677 \mathrm{GHz})$. All fibers are standard single mode fiber but the ones used as nonlinear medium. An optical circulator $\left(\mathrm{OC}_{1}\right)$ directs Pump\#1 toward the circular retarder, made of 1-km highly nonlinear fiber (HNLF) with $A_{e f f}=11 \mu \mathrm{m}^{2}, \Delta v_{B} \cong 50 \mathrm{MHz}, v_{B}=9.642 \mathrm{GHz}$ at $1548 \mathrm{~nm}$. Another optical circulator $\left(\mathrm{OC}_{2}\right)$ is used to inject Pump\#2 to the linear retarder, where the nonlinear medium is another HLNF. A fiber Bragg grating (FBG) in reflection mode (bandwidth of $12.5 \mathrm{GHz}$ ) is used to filter out backward residual pump waves. The total insertion loss of the system is $9 \mathrm{~dB}$. Polarization rotation is done by adjusting the pump power through the VOAs.

Figure 3a shows the evolution of the measured retardance with pump power as well as the output DOP at the output of the circular retarder. Each point in the figure is the average of 100 measurements with a maximum standard deviation of 0.02 and 0.45 for retardance and DOP, 
respectively. The retardance has been measured using the Poincaré sphere method [25]. It can be observed that, as given by (2), retardance varies linearly with pump power. The effect of the power of the input power on the induced retardance is small. When input signals of different power (up to $0.6 \mathrm{~mW}$ ) are rotated, the maximum standard deviation of the retardance was $4.6^{\circ}$. More information is provided in Annex A. Figure $3 \mathrm{~b}$ shows the evolution of the signal SOP at the output of the circular retarder when the power of Pump\#1 is changed from $0.08 \mathrm{~mW}$ to 12.6 $\mathrm{mW}$. It can be seen that a full rotation $(2 \pi)$ on the equator of the Poincaré sphere can be achieved.

However, there is no need for a full $2 \pi$ retardance change since the sense of the rotation on the Poincaré sphere can be tuned by swapping frequencies between $f_{p 1}$ and $f_{p 2}$. Thus, complete rotation of the SOP can be achieved, as shown in Figure 3c. For a retardance smaller than $\pi$, the DOP remains above $98 \%$.

a

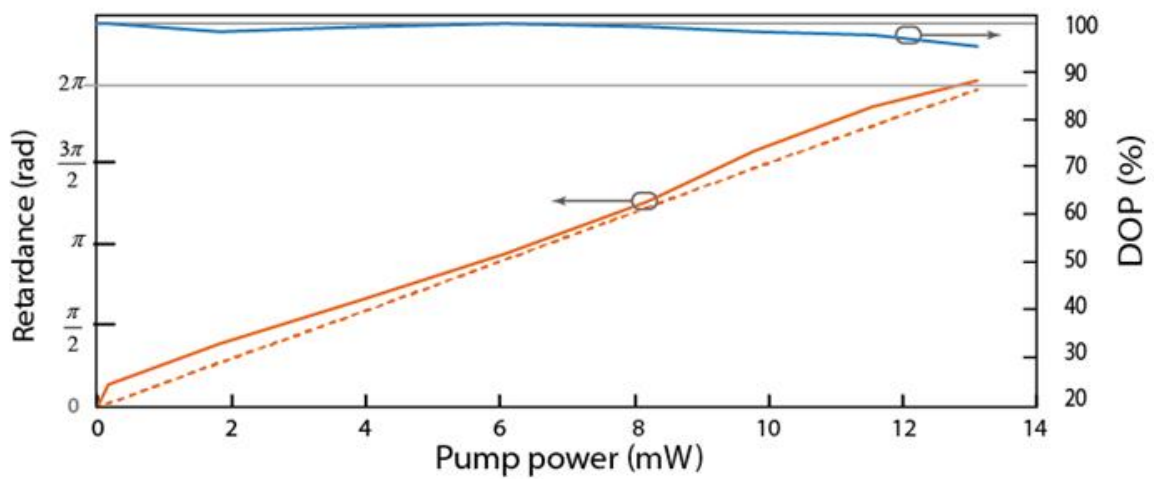

b

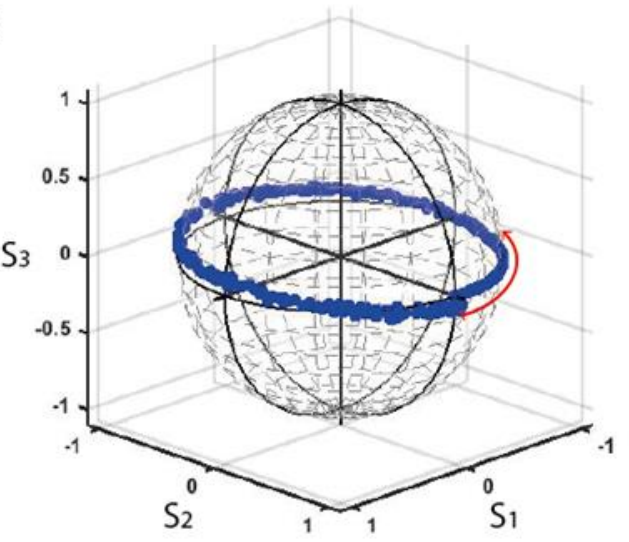

C

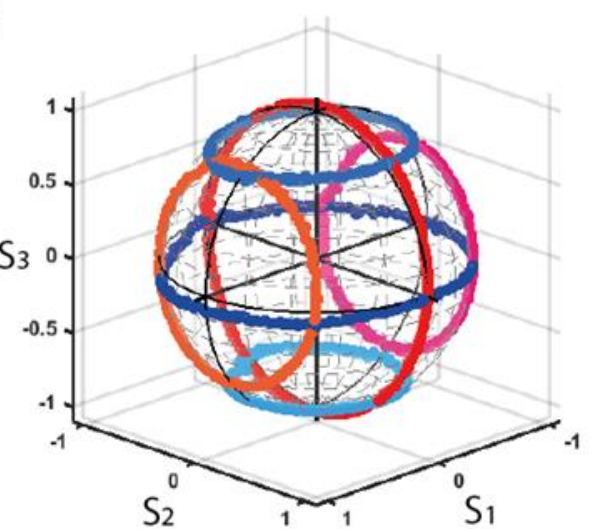

Fig. 3. Experimental results for a $0.16 \mathrm{~mW}$ input signal with linear polarization at $45^{\circ}$ (Jones vector $\left.\left([1 / \sqrt{2}(1,1)]^{T}\right)\right)$. (a) (Left) Retardance induced as a function of pump power for the circular retarder: experiment (solid orange) and theory (dotted orange); (Right) Evolution of the signal Degree of Polarization (DOP) for the circular retarder a function of pump power. (b) Poincaré sphere representation of the evolution of the output signal polarization over a circular retarder for an input signal. (c) Rotation of the signal SOP induced by the Brillouin-based circular and linear retarders.

Due to the combination of gain and loss responses, Brillouin polarization rotation induces only slight changes on the signal power in the range of pump powers that are needed to reach any point on the Poincaré sphere. Figure 4a shows measurements of the SBS-induced 
retardance for an input signal of $0.08 \mathrm{~mW}$ as a function of pump power and the variation of the power of the signal for the circular (blue solid and dotted) and linear (red solid and dotted) retarders. Figure 4a shows that the nonlinear variable retarder is able to provide different kinds of birefringence with the same efficiency. The same pump power induces the same retardance for the linear and the circular retarder. In addition, there are not preferred SOPs and the same pump power induce the same rotation angle on Poincare sphere independently of the input signal polarization state, as shown in Figure $4 \mathrm{~b}$.

The power of the signal experiences only a slight variation when it is rotated whereas the retardance grows with pump power, as it can also be seen in Figure 4a, which follows a trend similar to the behavior of the SBS gain. In particular, for small signals the variation of the insertion loss is smaller than $1.2 \mathrm{~dB}$. However, with signals that cannot be assumed to be weak in comparison to Brillouin threshold, the insertion loss increases_and, thus, the nonlinear polarization controller is limited to signals with power levels below it. For example, a variation of $2.5 \mathrm{~dB}$ is experienced for signals of $0.16 \mathrm{~mW}$ when rotated $2 \pi$. Further details are provided in Annex A.

As in other applications of SBS, the bandwidth of the Brillouin response can be tailored. Since Brillouin response is the convolution of the natural Brillouin gain spectrum with the normalized pump power spectrum, arbitrary polarization responses can be achieved by engineering the pump spectrum. Figure $4 \mathrm{c}$ shows the frequency dependence of the retardance induced by the Brillouin natural response (blue) and a flat-top retardance obtained with a multitone pump (red), which shows a maximum ripple of $2^{\circ}$. Broadened flat-top Brillouin responses enable control of signals from independent free-running sources, as done, for example, in [10]. It also allows the rotation of signals with bandwidths of the order or wider than the natural Brillouin response without partially depolarizing them.
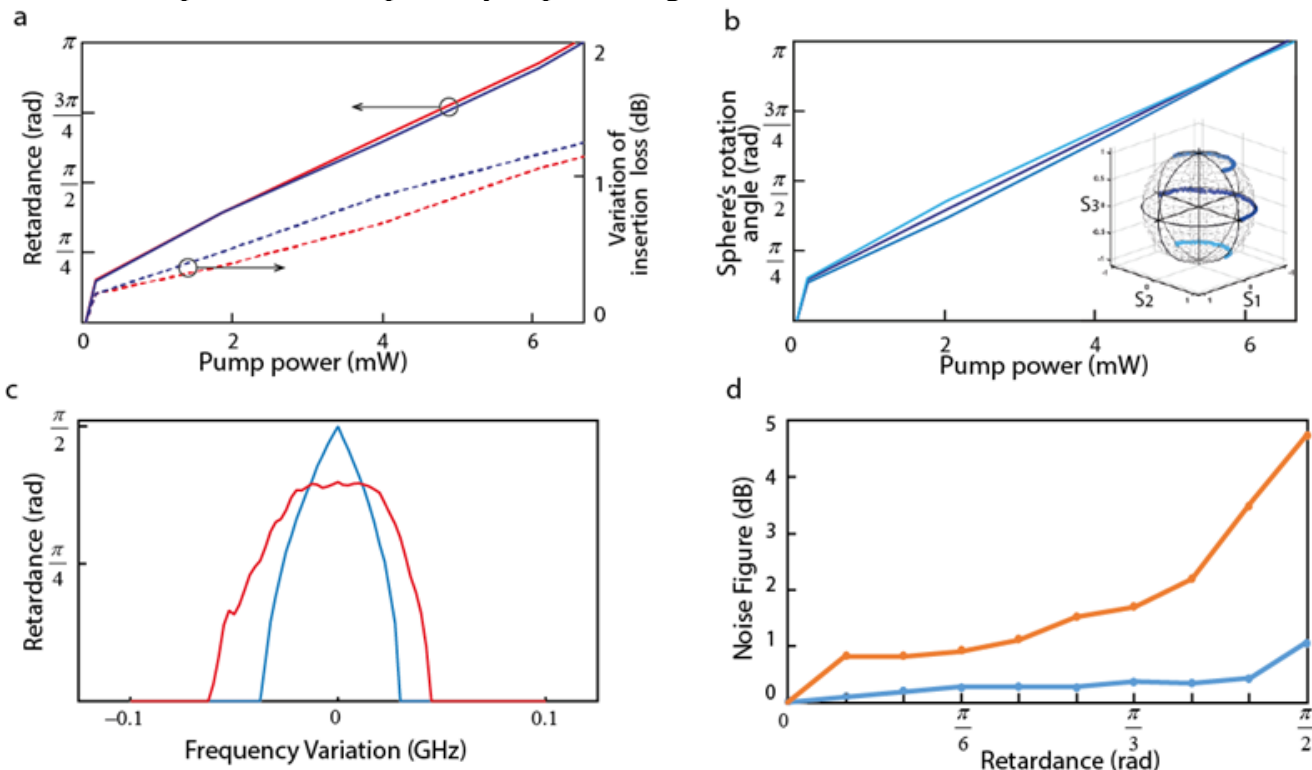

d

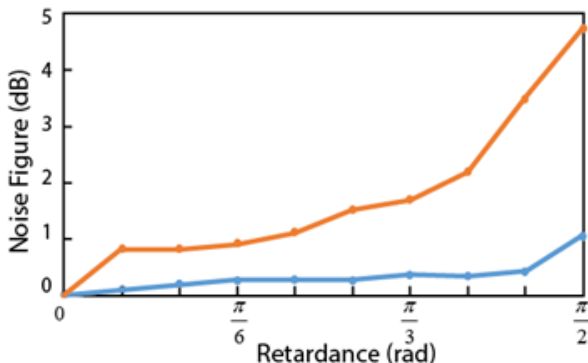

Fig. 4. . (a) (Left) Retardance induced as a function of pump power for the circular (blue solid) and linear (red solid) retarder for input signal with linear SOP at $45^{\circ}$ (Jones vector $\left([1 / \sqrt{2}(1,1)]^{T}\right)$ ). (Right) Variation of the output signal power versus pump power. (b) Sphere's rotation angle for input signal with ellipticity of \pm 0.5 (Jones vector $\left.\left[1 / \sqrt{2}\left(1, e^{\frac{j \pi}{4}}\right)\right]^{T},\left[1 / \sqrt{2}\left(1, e^{-\frac{j \pi}{4}}\right)\right]^{T}\right)$. (c) Control of the retardance bandwidth. (blue) Single pump configuration. (red) Multi-tone pump configuration. (d) Comparison of the change of the Noise Figure for the proposed method (blue) and polarization pulling (orange). 
Another interesting feature of the presented nonlinear polarization controller is that, unlike most Brillouin processing schemes, it introduces little additional noise due to the lack of net gain. To assess the noise addition by the polarization controller, the noise figure (NF) is defined as the ratio of SNR of the optical signal when the controller is off and on. Noise figure in both cases has been measured using an electrical method [25] (see additional details in Annex C). The change of the measured NF for a rotation between linear at 0 and $2 \pi$ has been measured showing a maximum variation of $1.5 \mathrm{~dB}$. For the sake of comparison, the proposed technique has been compared to polarization pulling, which induces a maximum pulling of $\pi / 2$. Given the gain induced by polarization pulling, the signal power for polarization pulling has been modified to produce the same RF beat note power. As it can be seen in Figure 4d, the polarization rotator experiences a NF variation of less than $1 \mathrm{~dB}$ with polarization pulling showing almost a $5 \mathrm{~dB}$ increment.

\section{Conclusion}

In this work, we have introduced a new all-optical functionality, nonlinear polarization control. Using stimulated Brillouin scattering, light polarization can be actively tailored, independently of adjacent signals. Experiments have shown that the full Poincare sphere can be reached while introducing optical power changes smaller than $2.5 \mathrm{~dB}$. Since Brillouin processing has the lowest power threshold among nonlinear effects in silica optical fibers, this effect can be easily harnessed. Thus, SBS can induce any type of birefringence with a single physical mechanism over the same material and with the same efficiency. The combination of gain and loss Brillouin responses avoids the generation of spontaneous Brillouin noise, which conventionally is the main limitation of Brillouin optical processing. This new functionality combined with its low noise feature opens new opportunities in polarization control from the synthesis of light with complex frequency-dependent polarization structures for chemistry, imaging and optical communications as well as a new approach to revisit some applications where Brillouin processing has been constrained by its noisy nature.

\section{Appendix A: Effect of changes of the input signal power}

Given the nonlinear nature of the polarization control method, the effect of the power of the input signal on the behavior of the polarization converter has been experimentally analyzed.

The effect of the power of the input signal on its output state of polarization (SOP) has been experimentally analyzed. Figure 5 shows, for the circular retarder, the retardance variation as a function of pump power for different input signal powers. It can be seen that the induced retardance is remarkably similar in all cases. A maximum change on retardance can be observed for a pump power of $8.3 \mathrm{~mW}$, with a standard deviation of the retardance of $4.6^{\circ}$. 


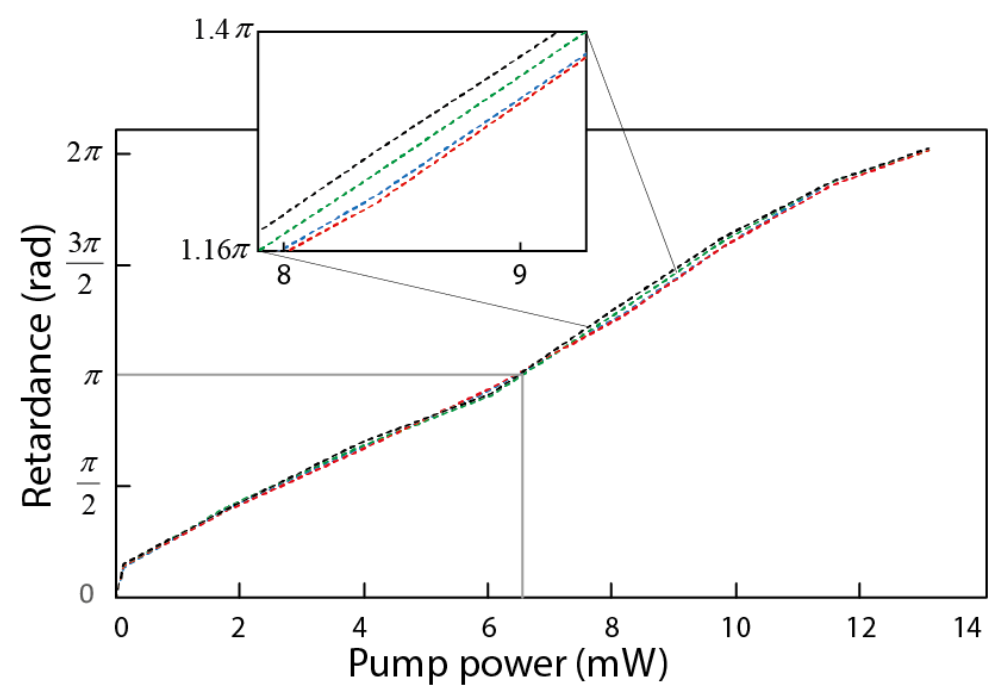

Fig. 5. Retardance versus pump power for a Brillouin circular retarder for signals of: $0.63 \mathrm{~mW}$ (dotted black), 0.16 $\mathrm{mW}$ (red dotted), $0.08 \mathrm{~mW}$ (dotted green) and $0.04 \mathrm{~mW}$ (dotted blue).

The effect of the power of the input signal on the loss of the polarization converter has also been analyzed. Figure 6 provides measurements of the converter insertion loss for different polarization conversions and signals with different input power. It can be seen that for input signals with moderate power (smaller than $0.16 \mathrm{~mW}$ ) and for pump powers smaller than $7 \mathrm{~mW}$, which induce retardance changes of $\pm \pi$, i.e. in the range of values of interest for polarization conversion, the maximum change of the insertion loss is $2.3 \mathrm{~dB}$. If the power of the input signal is high enough, the Brillouin process saturates and appreciable changes on the insertion loss of the polarization controller can be seen.

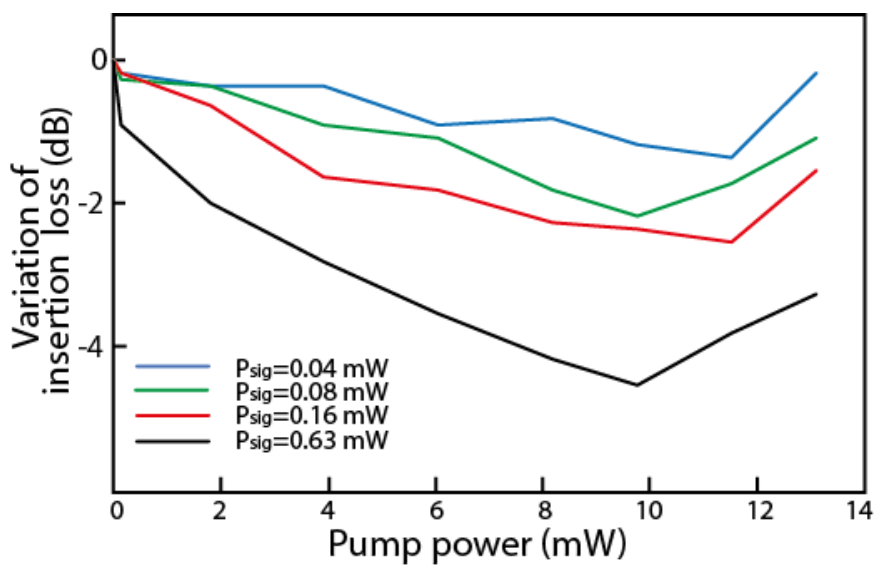

Fig. 6. Variation of the insertion loss of the polarization controller as a function of pump power for signals with different input power at the HLNF.

\section{Appendix B: Stability of the SOP}

Figure 7(a) show the temporal evolution of the Stokes vector components for a signal with input power at the HLNF of $0.16 \mathrm{~mW}$ and linear polarization at $45^{\circ}$ that is rotated with the Brillouin polarization controller to a linear signal at $135^{\circ}$, as depicted in Figure 7(b). 
(a)

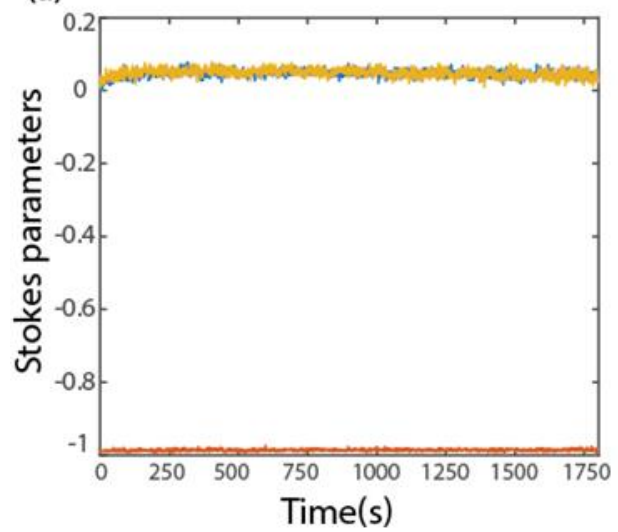

(b)

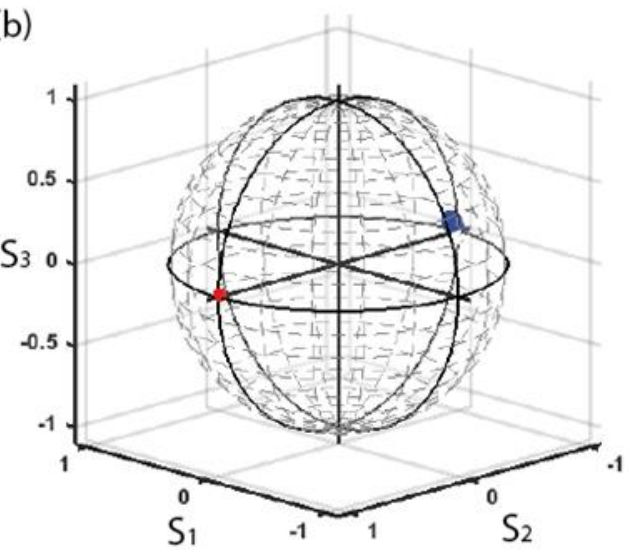

Fig. 7. Temporal stability of the signal SOP at the output of the polarization controller. (a) Stokes parameters of the signal output with linear SOP at $135^{\circ}$ as a function of time: (Color online) S1 (blue solid curve), S2 (red solid curve) and S3 (yellow solid curve). Stokes parameters are normalized to S0. (b) (Red) SOP of the signal without SBS, (blue) Signal SOP with SBS polarization conversion

The standard deviation of the Stokes parameters of the signal polarization with and without SBS polarization conversion is shown in Table 1.

\begin{tabular}{|c|c|c|c|}
\hline & S1 & S2 & S3 \\
\hline Rotated signal SOP & $8.3 \times 10^{-3}$ & $3.0 \times 10^{-3}$ & $9.9 \times 10^{-3}$ \\
\hline Unrotated signal SOP & $1.3 \times 10^{-3}$ & $0.6 \times 10^{-3}$ & $1.1 \times 10^{-3}$ \\
\hline
\end{tabular}

Table. 1. Standard deviation of Stokes parameters from Fig. S2 (a)

\section{Appendix C: Noise measurement}

The degradation on the Signal to Noise (SNR) ratio of the optical signal introduced by the Brillouin-based polarization converter, i.e. the noise figure (NF) of the nonlinear polarization converter, has been estimated. The NF has been defined as the degradation in SNR induced by the additional noise introduced by the polarization rotation. An electrical method to measure $\mathrm{NF}$ has been employed since it provides a more complete measure of the intensity noise generated by the controller [25]. Noise has been measured using the setup shown in Figure 8 where an RF signal $(2.5 \mathrm{GHz})$ has been used to modulate the optical signal. After heterodyne detection, the SNR of the beat note has been measured with an electrical spectrum analyzer. 


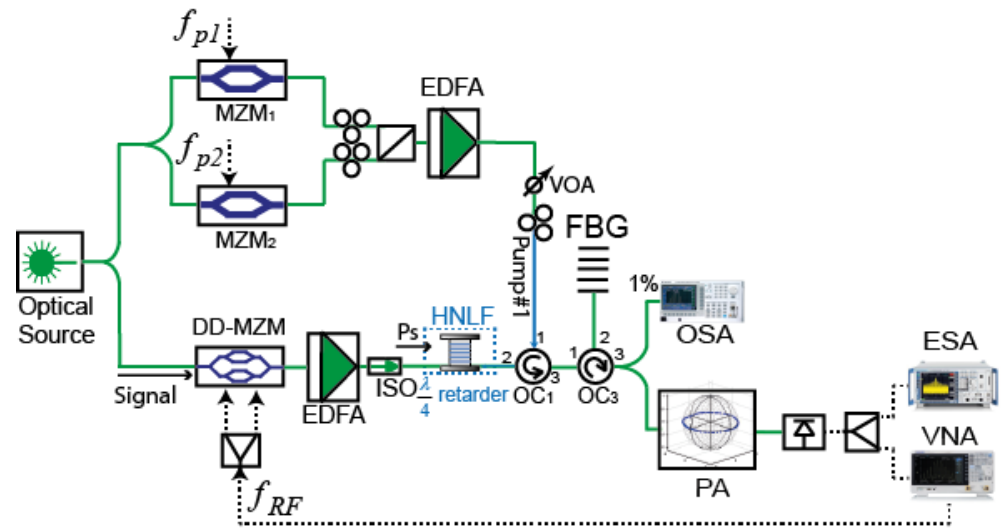

Fig. 8. Block diagram of a nonlinear all-optical polarization controller used to measure noise. HLNF: Highly nonlinear fiber. OC: Optical circulator; ISO: Isolator. VOA: Variable Optical Attenuator. FBG: Fiber Bragg grating. OSA: Optical spectrum analyzer. PA: Polarization analyzer. ESA: electrical spectrum analyzer. VNA: vector network analyzer.

The Brillouin-induced noise $\left(S_{P}(f)\right)$ must be separated from the total measured noise $\left(\left.S_{E S A}(f)\right|_{\text {mea }}\right)$ which also includes receiver thermal noise $\left(\left.S_{E S A}(f)\right|_{t h}\right)$, detected shot noise $\left(2 q i_{m e a}\right)$ and the frequency response of the system [25]. The thermal noise correction has been performed from measurement of the thermal noise density with the input light blocked. The correction of the system transfer function has been performed using a RIN transfer calibration setup. Finally, the shot noise has been corrected from measurements of the optical and electrical spectral of the amplitude modulated optical signal and its associated microwave beat note, respectively.

The intensity noise density produced by the polarization controller $\left(S_{p}(f)\right)$ has been derived from [25]:

$$
S_{P}(f)=\frac{\left.S_{E S A}(f)\right|_{m e a}-\left.S_{E S A}(f)\right|_{t h}}{\frac{\left.S_{E S A}(f)\right|_{c a l}}{R I N_{c a l}(f) P_{c a l}^{2}}}-\frac{2 q i_{\text {mea }}}{\mathfrak{R}^{2}}\left[\frac{W^{2}}{H z}\right]
$$

Then, the noise figure is [25]

$$
N F=\frac{P_{S} S_{P}(f)+2<G P_{S}>h v}{2 h v<G P_{S}>^{2}}
$$

where the gain $\left(G^{2}\right)$ has been derived from spectral analysis of the optical receiver photocurrent and $P_{s}$ is the power at the fiber input.

Funding. Spanish Ministry of Science, Innovation and Universities (TEC2016-80906-R), Republic of Ecuador SENESCYT (AR2Q-4233).

Disclosures. The authors declare no conflicts of interest.

\section{References}

1. Rogers, A. Polarization in Optical Fibers, (Artech House, 2008).

2. Wang, X.L., Luo, Y.H., Huang, H.L., Chen, M.C., Su, Z.E., Chao Chen, C.L., Li, W., Fang, Y.Q., Jiang, X., Zhang, J., Li, L. Liu, N.L., Lu, C.Y., Pan, J.W. 18-Qubit Entanglement with Six Photonic' Three Degrees of Freedom. Phys. Rev. Lett. 120, 26, 26502 (2018). 
3. Preece, D., Keen, S., Botvinick, E., Bowman, R., Padgett, M., Leach, J. Independent polarisation control of multiple optical traps. Optics Express. 16, 20, 15897-15902 (2008).

4. Heismann, F., Whalen, M. S. Broadband reset-free automatic polarisation controller. Electronics letters. 27, 4 377-379 (1991).

5. Koch, B., Noé, R., Mirvoda, V., Sandel, D., "100-krad/s endless polarisation tracking with miniaturised module card," Electron. Lett. 47, 813-814 (2011).

6. Yao, X.S. Fiber squeezer polarization controller with low activation loss. US Patent, US6480637, (2000).

7. Yao, X.S. Fiber devices based on fiber squeezer polarization controllers, US Patent, US6493474, (2002).

8. Zadok, A., Zilka, E., Eyal, A., Thevenaz, L. \& Tur, M. Vector analysis of stimulated Brillouin scattering amplification in standard single-mode fibers. Opt. Express. 16, 21602-21707 (2008).

9. Shmilovitch, Z., Primerov, N., Zadok, A., Eyal, A., Chin, S., Thevenaz, L., Tur, M. Dual-pump push-pull polarization control using stimulated Brillouin scattering. Opt. Express. 19, 25873-25880 (2011).

10. Vidal, B. Photonic millimeter-wave frequency multiplication based on cascaded four-wave mixing and polarization pulling. Opt. Lett. 37, 5055-5057, (2012).

11. Martinelli, M., Cirigliano, M., Ferrario, M., Marazzi, L. \& Martelli, P. Evidence of Raman-induced polarization pulling. Opt. Express. 17, 947-955 (2009).

12. Stiller, B., Morin, P., Nguyen, D.M., Fatome, J., Pitois, S., Lantz, E., Maillote, H., Menyuk, C.R., Sylvestre, T., "Demonstration of polarization pulling using a fiber-optic parametric amplifier," Opt. Express 20, 27248-27253 (2012).

13. Heebner, J.E., Bennink, R.S., Boyd, R.W. \& Fisher, R.A. Conversion of unpolarized light to polarized light with greater than $50 \%$ efficiency by photoredractiive two-beam coupling. Opt. Lett. 25, 257-259 (2000).

14. Kozlov, V.V., Nuño, J. \& Wabnitz, S. Theory of lossless polarization attraction in telecommunication fibers. J. Opt. Soc. Am. B. 29, 153-154 (2012).

15. Pitois, S., Millot, G. \& Wabnitz, S., Nonlinear polarization dynamics of counterpropagating waves in an isotropic optical fiber: theory and experiments. I. Opt. Soc. Am. B. 18, 432-443 (2001).

16. Fatome, J., Pitois, S., Morin, P. \& Millot, G. Observation of light-by-light polarization control and stabilization in optical fibre for telecommunication applications. Optics Express. 18, 15, 15311-15317 (2010).

17. Kozlov, V.V., Barozzi, M., Vannucci, A. \& Wabnitz, S. Lossless polarization attraction of copropagating beams in telecom fibers. J. Opt. Soc. Am. B. 30, 530-540 (2013).

18. DeLong, A., Astar, W., Mahmood, T., Carter, G.M., "Polarization attraction of 10-Gb/s NRZ-BPSK signal in a highly nonlinear fiber," Opt. Express 25, 25625-25636 (2017)

19. Loayssa, A. \& Lahoz, F.J. Broad-band RF photonic phase shifter based on stimulated Brillouin scattering and single-sideband modulation. IEEE Photonics Technology Letters. 18, 1, 208-210 (2006).

20. Pagani, M., Marpaung, D., Choi, D.Y., Madden, S.J., Luther-Davies, B., Eggleton, B.J. Tunable wideband microwave photonic phase shifter using on-chip stimulated Brillouin scattering. Optics express. 22, 23, 2881028818 (2014).

21. Galtarossa, A., Palmieri, L., Santagiustina, M., Chenato, L., Ursini, L., "Polarized Brillouin amplification in randomly birefringent and unidirectionally spun fibers," IEEE Photon. Technol. Lett. 20, 1420-1422 (2008)

22. Liao, M., Chaudhari, C., Qin, G., Yan, X., Kito, C., Suzuki, T., Ohishi, Y., Matsumoto, M., Misumi, T. Fabrication and characterization of a chalcogenide tellurite composite microstructure fiber with high nonlinearity. Opt. Express. 17, 21608-21614 (2009).

23. Morrison, B., Casas-Bedoya, A., Ren, G., Vu, K., Liu, Y., Zarifi, A., Nguyen, T.G., Choi, D.Y., Marpaung, D., Madden, S.J., Mitchell, A., Eggleton, B.J. Compact Brillouin devices through hybrid integration on silicon. Optica. 4, 847-853 (2017).

24. Chen, L, X. Bao, Analytical and numerical solutions for steady state stimulated Brillouin scattering in a singlemode fiber, Optics Communications, 152 (1-3), 65-70, 1998.

25. Derickson, D. Fiber Optica Test and Measurement. Ch. 6, 13 (Ed. Prentice Hall, 1998). 